\title{
Analysis of Gaucher Disease Responsible Genes in Colorectal Adenocarcinoma
}

\author{
Mesut Karatas ${ }^{1}$, Yusuf Turan ${ }^{2}$, Amina Kurtovic-Kozaric $^{1}$ and Şenol Dogan ${ }^{1^{*}}$ \\ ${ }^{1}$ Department of Genetics and Bioengineering, Faculty of Engineering and Information Technologies, International Burch University, Sarajevo, Bosnia and Herzegovina \\ ${ }^{2}$ Department of Biology, Faculty of Art and Science, Balikesir University,Balıkesir, Turkey
}

"Corresponding author: Şenol Dogan, PhD. Department of Genetics and Bioengineering, International Burch University, Sarajevo, Francuske revolucije bb, llidža, 71000 Sarajevo, Bosnia and Herzegovina, Tel: +387 33944 400; Fax: +387 33944 500; E-mail: senol1dogan3@gmail.com

Rec date: June 23, 2016; Acc date: June 25, 2016; Pub date: June 30, 2016

Copyright: ( 2016 , Karatas M, et al. This is an open-access article distributed under the terms of the Creative Commons Attribution License, which permits unrestricted use, distribution, and reproduction in any medium, provided the original author and source are credited.

\begin{abstract}
Gaucher disease is a hereditary genetic abnormality which defects the pathway of sphingolipid catabolism. The mutation of GBA gene which encodes lysosomal $\beta$-glucosidase enzyme is the main characteristics of the disease also is observed in different cancer types. To find the relation between the disease and colon adenocarcinoma, the responsible gene expression of Gaucher disease was analyzed. The gene expression of colon adenocarcinoma was compared between death and alive patients and analyzed statistically to profile the differences between Gaucher disease genes expression changes. GBA, GBA2, GBA3, SCARB2 and PSAP have the maximum genetic alteration which is observed in colon adenocarcinoma.
\end{abstract}

Keywords: Gaucher disease; Colon adenocarcinoma; Glucosphingolipid; Genetic mutations; Macrophages; Immune dysregulation

\section{Introduction}

Gaucher disease (GD) is an autosomal recessive inborn error in the pathway of sphingolipid catabolism. Biochemically, this disease is characterized by a deficiency of lysosomal $\beta$-glucosidase (glucocerebrosidase, GCase) enzyme due to the mutation of GBA gene [1]. The natural substrate of this lysosomal enzyme is glucocerebroside (also called glycosylceramide) mainly present on the cytoplasmic face of cellular membranes and on the cell surface. Lysosomal $\beta$ glucosidases play a key role in recycling of this substrate by degradation of glucosphingolipid from membranes of apoptatic white blood cells and senescent red blood cells. Genetic mutations of GBA gene affect the enzyme's catalytic activity, stability and result in the storage of hydrophobic glucocerebroside in the lysosomes of macrophage cells. The consequent accumulation of glucocerebroside in macrophages throughout the body leads to enlargement of the spleen and liver, skeletal disease and immune dysregulation [2]. The mutation of PSAP gene that encodes activator protein saposin C (enhances the activity of GCase) may cause gaucher disease rarely [3,4]. Gaucher disease can be classified into three types depending on the presence or absence of neurological features; Type I GD1 - adult or visceral form is characterized by the lack of central nervous system (CNS) involvement and accounts for almost 95\% of GD; Type II GD2 - infantile progressive neuropathic form with a life expectancy of $<2$ years; Type III (GD3) - juvenile, subacute neuropathic form [5]. Pathologically, patients with the adult non-neuropathic form of the disease have deposition of glucocerebroside in lysosomes of reticuloendothelial cells, particularly in the spleen, bone marrow and liver and frequently there is hepatosplenomegaly, anemia, and thrombocytopenia. It has been suggested that these tissue abnormalities in some Gaucher patients may trigger macrophage activation and could result in the enhanced inflammatory agents secretion and had increased levels of pro-inflammatory (i.e., TNF $\alpha$, IL-6, IL-8, and IL-1b) and antiinflammatory cytokines (i.e., CD14) in serum and/ or tissues [6-13]. TNF a production has been suggested as a response to glucosylceramide accumulation in Gaucher disease patients (a macrophage activation marker [2]. It has been reported that the immune dysregulation, aberrant macrophage activation and inflammatory imbalances increase the incidence of malignant disorders in patients Gaucher disease Type 1 (GD1) [2,6,7]. In 1982, Lee reported that 19 of the 35 deaths that occurred due to malignant disease among 239 patients with typel Gaucher disease; 3 of the patients had a diagnosis of multiple myeloma [6]. In 2005, Rosenbloom et.al reported cancer incidence among 2742 with patients Gaucher disease, using International Gaucher Registry they found that 10 of patients had multiple myeloma were diagnosed after the age 50 years [2].

How the insufficiency of GCase activity and the subsequent metabolic disturbances related to glucosylceramide and other sphingolipids could lead to such inflammatory imbalances remains unclear. Inflamation is thought to play a critical role in the advancement of cancer, particularly colorectal cancer [8]. As indicated by the reports, the processing of meat, by adding preservatives such as salt or sodium nitrite to prevent the growth of germs or smoking the meat to preserve or enhance color and flavor, may add compounds that might increase the potential of these foods to cause cancer. Studies have linked high intake of red meat, and particularly of processed meats with an increased risk of colorectal cancer [9]. In order to determine the relation of inflamatory inbalances and cancer development in patient with Gaucher disease and healthy people. We studied 10 genes.Inflammatory agents could be common factor for increasing the risk of cancer in both Gaucher patients and normal colorectal cancer patients. To better understand how the inflammatory imbalances lead to cancer in gaucher patient and normal human, we selected 10 genes which are GBA (encoding lysosomal glucocerebrosidase enzyme), GBA2, (encoding non-lysosomal glucosylceramidase enzyme) GBA3 (encoding cytosolic beta- 
Page 2 of 3

glucosidase enzyme), SCARB2 (encodes lysosomal integral membrane protein type 2 (LIMP-2) responsible for the transport of Gcase to the lysosome), PSAP (The PSAP gene (encoding saposin $\mathrm{C}$ which is an activator of glucocerebrosidase) IL1A, TNF, IL6, IL8, and LDHA.

\section{Materials and Methods}

\section{Materials}

Gene expression genomic data was the main type of material for the research. In addition to that, clinical data also has been used to find the patients ( $\mathrm{n}=18$ dead), ( $\mathrm{n}=163$ alive). The all data, gene expression and clinical data, have been downloaded from TCGA as UNCAgilent450, Agilent Expression 244K microarrays, COAD cancer type. In addition to that, control gene expression data have been used to compare the differences of the gene expression.

\section{Methods}

First of all the clinical data have been separated into dead and alive patients. The gene expression of the dead and alive patients also has been designed for them to measure the target genes, which could be effective in colon cancer, GBA, GBA2, GBA3, SCARB2, PSAP, IL1A, TNF, IL6, IL8, LDHA. The main idea of the method is to compare the target genes between dead and alive patients. t-test has been applied to the gene expression values to find the statistically significant P-value between dead and alive dataset [14-15].

\section{Results}

The target genes were all applied to statistical calculation to find significant changes. The genes were separated into two groups, colon cancer dead and colon cancer alive patients. The first analysis is the comparison between the cancer and control value. The target genes are generally high expressed in dead colon cancer patients, except GBA and LDHA low expressed ones -1.71 and -3.36 respectively. Then, the genes firstly were compared with control gene expression in healthy cell to see the differences between the cancer and control. $P$ value of the GBA, GBA3, SCARB2, PSAP, IL1A, TNF, IL6 and LDHA were statistically significant (Table 1).

\begin{tabular}{|l|l|l|l|l|}
\hline & $\begin{array}{l}\text { Cancer } \\
\text { Death }\end{array}$ & Control & Fold & P value \\
\hline GBA & -0.22 & 0.13 & -1.71 & 0.05 \\
\hline GBA2 & -0.4 & -0.65 & 0.61 & 0.061 \\
\hline GBA3 & 3.25 & 0.52 & 6.21 & 0.051 \\
\hline SCARB2 & 0.44 & 1.37 & 0.32 & 0.01 \\
\hline PSAP & 0.15 & 0.65 & 0.23 & 0.015 \\
\hline IL1A & 0.21 & 3.19 & 0.06 & 0.016 \\
\hline TNF & 1.34 & 1.4 & 0.96 & 0.045 \\
\hline IL6 & 0.68 & 5.66 & 0.12 & 0.029 \\
\hline IL8 & 0.25 & 4.19 & 0.06 & 0.108 \\
\hline LDHA & -1.45 & 0.43 & -3.36 & 0.05 \\
\hline
\end{tabular}

$P$ value of the gray ones is statistically significant genes

Table 1: The target gene colon cancer (Dead) gene expression.

Almost the similar result has been got from the target genes canceralive expression. While GBA and LDHA have low expressed the others show higher expressed profile. In addition to that, $p$ value of SCARB2, PSAP, IL1A, TNF, IL6 and LDHA genes were statistically significant (Table 2).

\begin{tabular}{|l|l|l|l|l|}
\hline & Cancer Alive & Control & Fold & P Value \\
\hline GBA & -0.28 & 0.13 & -2.12 & 0.063 \\
\hline GBA2 & -0.36 & -0.65 & 0.54 & 0.07 \\
\hline GBA3 & 3.09 & 0.52 & 5.9 & 0.065 \\
\hline SCARB2 & 0.58 & 1.37 & 0.42 & 0.016 \\
\hline PSAP & 0.29 & 0.65 & 0.45 & 0.022 \\
\hline IL1A & 0.41 & 3.19 & 0.13 & 0.023 \\
\hline TNF & 1.62 & 1.4 & 1.16 & 0.057 \\
\hline IL6 & 0.33 & 5.66 & 0.06 & 0.036 \\
\hline IL8 & 0.38 & 4.19 & 0.09 & 0.112 \\
\hline LDHA & -1.34 & 0.43 & -3.11 & 0.059 \\
\hline P value of the gray ones is statistically significant genes & \\
\hline
\end{tabular}

Table 2: The target gene colon cancer (Alive) gene expression.

The target genes were statistically analyzed by comparison deadcontrol and alive-control separately. Then, the t-test results show that both groups have statistically significant $\mathrm{p}$ value (Table 3 ).

\begin{tabular}{|l|l|l|}
\hline & P value & Alive/Control \\
\hline GBA & 0.01678 & $7.73 \mathrm{E}-07$ \\
\hline GBA2 & 0.00524 & $1.06 \mathrm{E}-05$ \\
\hline GBA3 & 0.38846 & $7.12 \mathrm{E}-12$ \\
\hline SCARB2 & $4.05 \mathrm{E}-09$ & $1.79 \mathrm{E}-21$ \\
\hline PSAP & 0.00016 & $2.86 \mathrm{E}-05$ \\
\hline IL1A & $2.62 \mathrm{E}-12$ & $2.13 \mathrm{E}-24$ \\
\hline TNF & 0.38161 & 0.01904 \\
\hline IL6 & $1.08 \mathrm{E}-27$ & $7.09 \mathrm{E}-53$ \\
\hline IL8 & $2.60 \mathrm{E}-11$ & $7.48 \mathrm{E}-22$ \\
\hline LDHA & $4.26 \mathrm{E}-10$ & $2.06 \mathrm{E}-40$ \\
\hline
\end{tabular}

Table 3: The $\mathrm{P}$ value comparison between death and alive cancer patients.

The Gaucher disease responsible genes genetic alteration is also tested by a genomic web tool (http://www.cbioportal.org/ 
data_sets.jsp). According to the analysis, GBA2 has the maximum genetic alteration \%2.9. The missense mutation mostly is observed in GBA2, SCARB2, IL6 and LDHA. While amplification and deletion are also observed in GBA, GBA2, PSAP, GBA3 shows the highest truncating mutation (Figure 1).

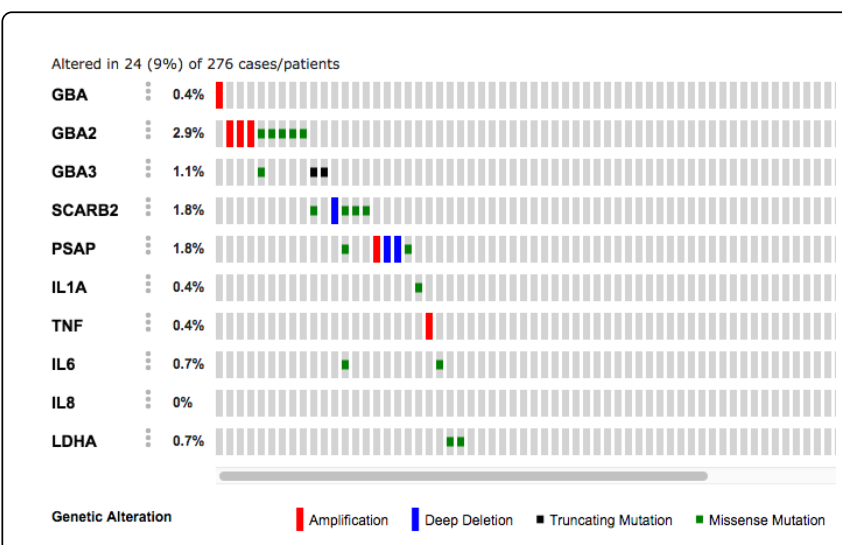

Figure 1: Genomic alteration of the target genes in colon adenocarcinoma.

\section{Discussion and Conclusion}

Colon cancer is one of the rising cancer types especially among the urban citizens. Food additives which are used for most of the industrialized food products could be harmful for changing epigenetics of human genome. Gaucher disease is a kind of some enzyme disabilities because of somatic mutation. It is already known that the disease is related to some other cancer types. Therefore, we decide to analyze the gene expression and genomic alteration of the genes which are responsible for Gaucher disease in colonoadrenocarcinoma. As a result of the analysis, the genes expression profile shows that they are genetically dynamic ones. In the future, the disease could be used as a signal to diagnose the cancer types.

\section{References}

1. Raghavan SS, Topol J, Kolodny EH (1980) Leukocyte beta-glucosidase in homozygotes and heterozygotes for Gaucher disease. Am J Hum Genet 32: 158-173.
2. Rosenbloom BE, Weinreb NJ, Zimran A, Kacena KA, Charrow J, et al. (2005) Gaucher disease and cancer incidence: a study from the Gaucher Registry. Blood 105: 4569-4572.

3. Tamargo RJ, Velayati A, Goldin E, Sidransky E (2012) The role of saposin C in Gaucher disease. Mol Genet Metab 106: 257-263.

4. Lieberman RL (2011) A Guided Tour of the Structural Biology of Gaucher Disease: Acid-1 $\hat{I}^{2}$-Glucosidase and Saposin C. Enzyme Res 2011: 973231.

5. Charrow J, Esplin JA, Gribble TJ, Kaplan P, Kolodny EH, et al. (1998) Gaucher disease: recommendations on diagnosis, evaluation, and monitoring. Arch Intern Med 158: 1754-1760.

6. Lee RE (1982) The pathology of Gaucher disease. Prog Clin Biol Res 95: 177-217.

7. Shiran A, Brenner B, Laor A, Tatarsky I (1993) Increased risk of cancer in patients with Gaucher disease. Cancer 72: 219-224.

8. Klampfer L (2011) Cytokines, inflammation and colon cancer. Curr Cancer Drug Targets 11: 451-464.

9. World Cancer Research Fund (2007) Food, nutrition, physical activity, and the prevention of cancer: A global perspective. American Institute of Cancer Research Washington, USA.

10. Barak V, Acker M, Nisman B, Kalickman I, Abrahamov A, et al. (1999) Cytokines in Gaucher's disease. Eur Cytokine Netw 10: 205-210.

11. Hollak CE, Evers L, Aerts JM, van Oers MH (1997) Elevated levels of MCSF, sCD14 and IL8 in type 1 Gaucher disease. Blood Cells Mol Dis 23: 201-212.

12. Michelin K, Wajner A, Bock H, Fachel A, Rosenberg R, et al. (2005) Biochemical properties of beta-glucosidase in leukocytes from patients and obligated heterozygotes for Gaucher disease carriers. Clin Chim Acta 362: 101-109.

13. Michelakakis H, Spanou C, Kondyli A, Dimitriou E, Van Weely S, et al. (1996) Plasma tumor necrosis factor-a (TNF-a) levels in Gaucher disease. Biochim Biophys Acta 1317: 219-222.

14. S Dogan, A Kurtovic-Kozaric (2016) The extremely high and low expressed genes by EGEF algorithm in invasive breast cancer. Journal of Biometrics \& Biostatistics.

15. S Dogan (2015) Detection of G-type density in promoter sequence of colon cancer oncogenes and tumor suppressor genes. Bioinformation 11: 290-295. 